\title{
Kompetenzen im Widerstreit
}

\section{Unternehmensberater als Personalplaner der Deutschen Bundespost}

I $983-1985$

von Alina Marktanner

Das Gewerkschaftsblatt „Die Deutsche Postgilde“ nahm im Februar I984 den Einsatz zweier Beratungsunternehmen bei der Deutschen Bundespost aufs Korn: „Belächelte man zunächst [...] die Interimcoachs [...], so empfindet man sie zunehmend als

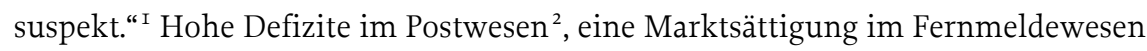
sowie die schwindende Unantastbarkeit des Berufsbeamtentums ${ }^{3}$ hatten sich bereits seit Mitte der vorhergehenden Dekade zu einer drückenden Problemlage verdichtet. Offenbar wollte der Minister für das Post- und Fernmeldewesen der ersten Kohl-Regierung, Christian Schwarz-Schilling, diese nicht allein bewältigen. Die „Coachs“, die er zur Rettung engagiert hatte, waren die Beratungsfirmen Knight Wendling und Mummert + Partner. Das Schweizer Unternehmen Knight Wendling hatte ein strategisches Unternehmenskonzept für die Deutsche Post auszuarbeiten, während die Hamburger Firma Mummert + Partner das Personalbemessungssystem auf Verbesserungsmöglichkeiten hin prüfen sollte. Der geballte Unmut der unteren Verwaltungsebenen gegen die Berater von, bissig apostrophiert, „Mumpitz und Wendelin“ mündete in einem Aufruf zum Boykott der Projekte: „Was liegt da näher, als mit probaten Spielmethoden den millionenschweren Neuerwerbungen Abseitsfallen zu bauen, sie abzublocken, ins Leere laufen zu lassen oder sich einfach totzustellen." 4

Die Beratungsaufträge der I980er Jahre bei der Deutschen Bundespost stehen bei-

\footnotetext{
I Mumpitz und Wendelin, in: Die Deutsche Postgilde I984, 2.

2 Bundesministerium für das Post- und Fernmeldewesen (Hrsg.), Deutsche Bundespost. Geschäftsbericht I980. Bonn I980, 86; Bundesministerium für das Post- und Fernmeldewesen (Hrsg.), Deutsche Bundespost. Geschäftsbericht I98I. Bonn I98I.

3 Mit seinem „Aktionsprogramm zur Dienstrechtsreform“ von I 976 führte der damalige Bundesinnenminister Werner Maihofer erstmals die Prinzipien der funktions- und leistungsgerechten Bezahlung in das öffentliche Dienstrecht ein. Vgl. Franz Kroppenstedt, Das Aktionsprogramm der Bundesregierung zur Reform des öffentlichen Dienstrechts, in: Die öffentliche Verwaltung I/2, I977, I56.
}

4 Mumpitz (wie Anm. I), 2. 
spielhaft für ein Phänomen der jüngeren deutschen Geschichte, das sich mit dem Begriff Behörden-Consulting fassen lässt: die Beratung von Bund, Ländern und Gemeinden sowie öffentlicher Unternehmen durch Unternehmensberatungsfirmen. ${ }^{5}$ Der Einsatz von Organisations- und Strategieberatungen in der staatlichen Verwaltung eröffnet eine Dichotomie öffentlicher und privater Logiken und ist am ehesten als Verquickung wechselseitiger Interessen zu verstehen. Unternehmensberater erschlossen mit staatlichen Kunden ein neues Marktsegment, während politische Entscheidungsträger ihr Tätig- oder Untätigsein mithilfe externer Gutachten abzusichern versuchten. Konnten sich Auftraggeber und Auftragnehmer auf eine erfolgreiche Zusammenarbeit einigen, was nicht immer gelang, erreichten interne Behördenkonflikte durch die äußere Kontrolle jedoch erst recht ihren Höhepunkt. Im Falle der Deutschen Bundespost stellte Schwarz-Schilling externe Beratergutachten als für ein öffentliches Unternehmen notwendig und zeitgemäß dar. Die Beamten und Angestellten der Post- und Fernmeldeämter lehnten die Consultants dagegen als bedrohliche Eindringlinge ab.

Der vorliegende Beitrag behandelt das Beratungsprojekt der Mummert + Partner Unternehmensberatung GmbH zum Personalbemessungssystem der Deutschen Bundespost in den Jahren I 983 bis I 985 und fragt: Wie kollidierten und wetteiferten unterschiedliche Wissensakteure und Interessensgemeinschaften im Streit um das Bemessungssystem der Deutschen Bundespost? Der Beitrag stützt sich auf Archivmaterial, zeitgenössische Veröffentlichungen der Verwaltungswissenschaften, einige Presseartikel sowie Zeitzeugengespräche mit ehemaligen Beratern. Im Zentrum steht eine spannungsgeladene Akteurskonstellation: Bundesminister SchwarzSchilling, der mit der Prüfung des Bemessungssystems darauf abzielte, Personalkosten zu sparen; die internen Bemessungsfachleute der Deutschen Bundespost, die ihre Kompetenz nicht in Frage gestellt sehen wollten; die Deutsche Postgewerkschaft, die Stellenstreichungen und Ansehensverlust der Belegschaft zu verhindern suchte; und die externen Berater, die durch die Gemengelage der Interessen navigier-

5 Siehe zum US-amerikanischen Fall Christopher D. McKenna, The World's Newest Profession. Management Consulting in the Twentieth Century. Cambridge 2006, 80-I I0; zum französischen Fall Philippe Bezes, Réinventer l'État. Les réformes de l'administration française (I962-2008). Paris 2009; vergleichend zu Kanada, Großbritannien und Frankreich Denis Saint-Martin, Building the New Managerialist State. Consultants and the Politics of Public Sector Reform in Comparative Perspective. Oxford 2000; kritisch aus soziologischer Perspektive: Peter Richter, Ökonomisierung als gesellschaftliche Entdifferenzierung. Eine Soziologie zum Wandel des öffentlichen Sektors. Konstanz 2009. 
ten und gemäß ihres Auftrages die Rationalisierungsbemühungen des Ministers als alternativlos präsentierten. Ihre jeweiligen Interessen versuchten die unterschiedlichen Parteien mit einem diskursiven Manöver durchzusetzen: der (Selbst-) Zuschreibung und Aberkennung von Expertise.

Die Beauftragung externer Berater, so zeigt der Beitrag, sollte die Postbeamten im Bemessungsbereich als voreingenommen und unternehmerischem Denken gegenüber verschlossen exponieren. Nachdem der Minister für das Post- und Fernmeldewesen die Prüfung seines eigenen Hauses veranlasst hatte, nahm die öffentliche Kritik an der Bundespost zu, und es entstand das Bild unfähiger und bequemer Postbeamter. Reibungsverluste für die Ministeriumsspitze ergaben sich dennoch, da die Belegschaft und ihre Sprachrohre weder bereit waren, den Consultants einen Expertenstatus zuzugestehen noch den eigenen beschneiden zu lassen. Indem Postgewerkschafter und Hauptpersonalräte die Gutachten von Unternehmensberatern als „fragwürdig“ und „praxisfremd“ abqualifizierten, versuchten sie ihrerseits, den Firmenvertretern ihre Kompetenz streitig zu machen und die eigene Deutungshoheit zu erhalten. ${ }^{6}$ Letztlich begünstigte die Autoritätsverschiebung von verwaltungseigenen Sachverständigen hin zu äußeren Kontrollinstanzen aber die ministeriellen Sparpläne.

Im Folgenden erläutere ich zunächst meinen Zugriff auf die Geschichte der Expertise als eine Geschichte von Selbst- und Fremdzuschreibungen. Danach zeichne ich nach, wie in der zweiten Hälfte des 20.Jahrhunderts das Bild des Unternehmensberaters als neutralem Außenstehenden in Wirtschaft und Verwaltung entstand. Im dritten Schritt analysiere ich den Vorlauf, die Durchführung und die Rezeption der Poststudie von Mummert + Partner I983 bis I985. Wichtige Knotenpunkte sind dabei Kompetenzstreitigkeiten, die posteigene Bemessungsfachleute sowie Gewerkschafter mit den Beratern suchten. Warum bröckelte der Expertenstatus der Beamten, und warum konnten sich Externe erfolgreich mit einem solchen schmücken?

6 Personalbemessung. Auf wackliger Grundlage, in: Deutsche Post, 20.6.I985, I 2. 


\section{Neue Anforderungen an externe Verwaltungsberater: Interessenvermittlung über Objektivität}

Studien der deutschen Zeitgeschichtsschreibung verstehen den Expertenbegriff als sozial und historisch geworden. Methodisch wird nicht von inhärent überlegenem Wissen ausgegangen, das Experten auf Organisationen „transferieren“. Stattdessen stellt sich die Frage, in welchen Akteurskonstellationen und Anwendungskontexten Expertenwissen entstand und welche Wechselwirkungen es in diesen entfaltete. ${ }^{7}$ Das Beispiel des Behörden-Consultings ist symptomatisch für den erhöhten Legitimationsdruck, dem sich die Bundesregierung, die Landesregierungen und ihre Verwaltungsspitzen angesichts immer größerer Haushaltsdefizite etwa ab Mitte der I970er Jahre ausgesetzt sahen. Mit der „großen Ernüchterung“ ${ }^{8}$ hinsichtlich keynesianischer Wirtschaftsprognosen und staatlicher Steuerungsfähigkeit keimte gleichzeitig das Interesse von Bundes- und Landesministern auf, ihre Häuser von kommerziellen Beratungsfirmen auf Organisationsmängel prüfen zu lassen. Ähnlich wie in der Privatwirtschaft etwa eine Dekade früher zeichnete sich im Gemeinwesen eine aufkommende „Unsicherheit in Bezug auf die angemessene Form von Organisation und Führung“ ab. ${ }^{9}$ Das verschärfte Gebot der Haushaltskonsolidierung stellte das Verhältnis der Sozialpartner auf die Probe und verlangte nach neuen Mechanismen der Vermittlung bei Interessenkonflikten. ${ }^{\text {Io }}$

Unternehmensberater versprachen dabei kein verlässlicheres Wissen als akademische Gremien, sondern „sozial robusteres“, wie es Peter Weingart und Kollegen formulieren. ${ }^{\text {II }}$ Die externen Berater griffen die Sichtweisen unterschiedlicher Konfliktparteien auf und ließen in widersprüchlichen Gemengelagen auf praxisrelevante Lösungsvorschläge hoffen. Als ihr Produkt galt die Fähigkeit, Organisationen

7 Siehe Ruth Rosenberger, Experten für Humankapital. Die Entdeckung des Personalmanagements in der Bundesrepublik Deutschland. München 2008, I 5 und die dort zitierte Literatur.

8 Tim Schanetzky, Die große Ernüchterung. Wirtschaftspolitik, Expertise und Gesellschaft in der Bundesrepublik I966 bis I982. Berlin 2007.

9 Werner Plumpe, Das kalte Herz. Kapitalismus: Die Geschichte einer andauernden Revolution. Berlin 20I9, 557 .

Io Zum Aufstieg der „Konsolidierungskoalition“ in Wissenschaft, Politik und Öffentlichkeit siehe HansPeter Ullmann, Das Abgleiten in den Schuldenstaat. Öffentliche Finanzen in der Bundesrepublik von den sechziger bis zu den achtziger Jahren. Göttingen 20I7, 275-300.

I I Peter Weingart/Martin Carrier/Wolfgang Krohn(Hrsg.), Nachrichten aus der Wissensgesellschaft. Analysen zur Veränderung der Wissenschaft. Weilerswist 2007, 299f. 
gleich welcher Art unter betriebswirtschaftlichen Gesichtspunkten zu analysieren und unter numerisch-quantitativen Aspekten aufzuschlüsseln. Gleichzeitig bedienten sie ein sich ausbreitendes „Fortschritts-, Modernisierungs- und Rationalisierungsparadigma“ “22 das ein neues, an ökonomischen Kennzahlen orientiertes Steuerungsvermögen versprach.

Ihre Dienste bewarben sie als sachlich und interessensbefreit, indem sie sich auf einen bestimmten Wissenstypus zurückzogen: den sogenannten „gesunden Menschenverstand“. Oft synonym gebraucht mit dem Begriff der Effizienz, verbarg sich hinter dem Konzept des gesunden Menschenverstandes eine Ansammlung betriebswirtschaftlicher Prinzipien. Indem sie ihre Unkenntnis organisationseigener Spezifika plakativ zur Schau stellten, versahen Unternehmensberater intern gewachsenes Wissen implizit mit dem Makel der déformation professionnelle. In der Behördenberatung rührten sie damit an so manches Tabu um wohlgehütete Schätze der Verwaltung, wie das Berufsbeamtentum, und lieferten Begründungsnarrative für diejenigen Entscheidungsträger, die einen kosteneffizienten und leistungsorientierten Staat herbeisehnten.

Die Gewerkschaften und Personalvertretungen der Deutschen Bundespost hatten Consultingfirmen wie McKinsey \& Company bereits Mitte der I $970 e r$ Jahre als Rationalisierungsbeauftragte fürchten gelernt. ${ }^{\mathrm{I}}$ Entsprechend hatten sie sich bereits nach Strategien gegen angeordnete Betriebsprüfungen umgehört. ${ }^{\mathrm{I} 4}$ Die Beratungsprojekte der I980er Jahre waren jedoch so umfassend angelegt, dass sie Pläne für eine Neuorganisation des öffentlichen Unternehmens nachhaltig zu befördern drohten. Besonders die Untersuchung der Firma Mummert + Partner zum Personalbemessungssystem wirkte in den Augen der Betroffenen wie ein Einfallstor für Stellenstreichungen und die gefürchtete Privatisierung. ${ }^{15}$ Dies begründete sich in Hinblick auf die Kritik, die sich seit Mitte der I97oer Jahre an der geringen „Effizienz“

\footnotetext{
I2 Andreas Wirsching, Abschied vom Provisorium I982-I99o. Geschichte der Bundesrepublik Deutschland. Stuttgart 2006, 44I.

I3 Siehe zum Beispiel McKinsey \& Company (Hrsg.), Möglichkeiten für eine Neuregelung der Busdienste von Deutscher Bundesbahn und Deutscher Bundespost. Düsseldorf I 976.

I4 Gefunden im Archiv der sozialen Demokratie, Bestand der Deutschen Postgewerkschaft: Gewerkschaft Handel, Banken und Versicherungen (Hrsg.), Arbeitsheft Rationalisierung für Betriebsräte, Personalräte und Vertrauensleute. Achtung - McKinsey kommt! Düsseldorf I 98r.

I5 Bis dahin sollte der Weg aber noch lang sein. Vgl. Thomas Handschuhmacher, „Was soll und kann der Staat noch leisten?" Eine politische Geschichte der Privatisierung in der Bundesrepublik I949-I 989. Göttingen 20I 8, 25I-297.
} 
der öffentlichen Verwaltung verdichtet hatte. ${ }^{16}$ Führende Verwaltungswissenschaftler und Organisationssoziologen, die selbst tragende Rollen in der Politikberatung bekleideten, kritisierten steigende Kosten insbesondere im öffentlichen Dienst und bemängelten eine gleichzeitig disproportionale „Produktivität“ der Behörden. ${ }^{17}$ Ihre Schriften verfestigten das Bild des „Selbsterhaltungsstrebens“ von Behörden, das zu überhöhten Forderungen nach Personal und Sachmitteln und damit zu unverhältnismäßigen Staatsausgaben führe. ${ }^{\text {I8 }}$

Das zwangsweise verstärkte Kostenbewusstsein in der öffentlichen Verwaltung richtete sich daher früh auf den Personalbestand in Behörden. In einer Studie zur „Personalbemessung in der Ministerialverwaltung“ für das Bundesministerium des Inneren beschrieben die Verwaltungswissenschaftler Siedentopf und Schmid Personalbemessung 1979 als Vorgehensweise, „die es erlaubt, die Zahl und Qualität der Arbeitskräfte zu ermitteln und festzulegen, die erforderlich sind, um räumlich und zeitlich bestimmte Aufgaben unter Beachtung betriebsspezifischer Eigenheiten zu erfüllen“. ${ }^{\text {I9 }}$ Eine kriteriengestützte Berechnung des Personalbedarfs sollte eine „sachgerechte personalwirtschaftliche Planung“ ermöglichen und für einen „rationellen Personaleinsatz in allen Arbeitsbereichen der betrieblichen Organisation“ sorgen. ${ }^{20}$ Siedentopf und Schmid stellten eine Auswahl an Bemessungsmethoden vor, die in privatwirtschaftlichen Unternehmen angewandt wurden, darunter statistische Erhebungen, Schätzverfahren und tayloristische Zeitstudien. ${ }^{2 \mathrm{I}}$

Diese Einblicke waren für die Bemessungsfachleute der Deutschen Bundespost nicht neu. Im Unterschied zu den meisten anderen staatlichen Organisationen stellte das öffentliche Unternehmen den Personalbedarf der Ämter schon seit Anbruch des 20. Jahrhunderts systematisch fest. ${ }^{22}$ Die Tatsache, dass der Postminister das Bemessungssystem I983 trotz dieses Wissensschatzes extern überprüfen ließ, zeugte

\footnotetext{
I6 Renate Mayntz, Soziologie der öffentlichen Verwaltung. 2.Aufl. Heidelberg I982, I 26.

I7 Volker Hauff/Fritz W. Scharpf, Modernisierung der Volkswirtschaft. Technologiepolitik als Strukturpolitik. Frankfurt am Main I975, I02.

I8 Mayntz, Soziologie (wie Anm. I6), I29; Thomas Ellwein/Joachim Jens Hesse, Das Regierungssystem der Bundesrepublik Deutschland. 6. Aufl. Opladen I987, 342.

I9 Heinrich Siedentopf/Karl-Rolf Schmid, Personalbemessung in der Ministerialverwaltung. Eine Vorstudie. (Speyerer Forschungsbericht, Bd. 6.) Speyer 1979, 2.

20 Ebd.

2 I Ebd.4-6.

22 Kurt Gscheidle, Die Personalbemessung bei der Deutschen Bundespost, in: ders. (Hrsg.), Jahrbuch der Deutschen Bundespost I982. Bad Windsheim I982, I 2.
} 
von einer sich weitenden Kluft zwischen Behördenspitze und Belegschaft. Aufgrund hoher Personalkosten musste „[j]eder Postminister, der schwarze Zahlen schreiben wollte, [...] Kostenbewusstsein im Personalbereich herstellen und durfte den Konflikt mit den mächtigen Personalräten und der Postgewerkschaft nicht scheuen““. ${ }^{23}$ Als Chef der größten Bundesbehörde mit mehr als 500000 Mitarbeitern hatte Schwarz-Schilling in der Tat zahlreiche interne Widerstände zu überwinden, wollte er auf den Stellenhaushalt einwirken. Dazu gehörten auch die für die Personalbemessung zuständigen Beamten und Angestellten. Die „viel beklagte ,Macht des ministeriellen Apparates“ “ hatte schon sein Vorgänger Kurt Gscheidle unterstrichen. ${ }^{24}$ Die fachliche Aufbereitung eines Themas durch die Referate bedingte die ministerielle Entscheidungsfindung bedeutend mit. Der politische Verantwortungsträger habe „meist nicht mehr die geringste Chance, sich eine eigene Meinung zu bilden und zu einem von der Entscheidungsempfehlung abweichenden Ergebnis zu kommen“. ${ }^{25}$ Schwarz-Schillings Beraterprojekte der I 980er Jahre waren ein Versuch, größere Souveränität über die Beamtenschaft zu erringen, einen künftig geringeren Stellenbedarf zu errechnen und so die Finanznot des Postwesens in den Griff zu bekommen.

\section{Unternehmensberater - Experten wofür?}

Unternehmensberater galten seit den Anfängen ihrer Branche im Deutschland der I920er Jahre ausdrücklich nicht als Fachexperten. In Selbst- und Fremddarstellungen erschienen sie als Transferdenker, die in der Lage seien, Schwachstellen von Organisationen in vergleichender Perspektive zu erkennen und zu bewerten. Das Bild des „unabhängigen Beraters“, dessen „Hilfe“ an Stellen gefragt sei, an denen „die Gefahr der Betriebsblindheit“ bestehe ${ }^{26}$, zeichnete zum Beispiel das Rationalisierungskuratorium der Deutschen Wirtschaft (RKW). Eine deutsche Delegation hatte

23 Werner Abelshauser, Nach dem Wirtschaftswunder. Der Gewerkschafter, Politiker und Unternehmer Hans Matthöfer. Bonn 2009, 536.

24 Kurt Gscheidle, Damit wir in Verbindung bleiben. Portrait der Deutschen Bundespost. Stuttgart-Degerloch i982, i i 9 .

25 Ebd.

26 Staatsarchiv Hamburg, I3I-I3/433, Kommunale Gemeinschaftsstelle für Verwaltungsvereinfachung, Ausschnitt aus den Mitteilungen der KGSt vom 25. Mai I966, Nr. Io. 
I953 in den USA mehrere Betriebsberatungsfirmen besucht und berichtete beeindruckt, dass Deutschland am „Beispiel der amerikanischen Wirtschaft“ noch viel lernen könne. ${ }^{27}$ Durch die Vielzahl seiner Aufträge und Vergleichsmöglichkeiten könne der Berater „wie ein Arzt an bestimmten, immer wiederkehrenden Symptomen vorhandene Fehlerquellen viel leichter und schneller diagnostizieren [...] als selbst der tüchtigste Manager“. ${ }^{28}$ In ihrer Absicht, das Beratungswesen in Deutschland zu stärken, hoben die Reisenden die Vorzüge einer Außen- gegenüber der betrieblichen Innenperspektive hervor. So „dürfte fast selbstverständlich sein, daß eine eingespielte Gruppe [...], die Erfahrungen in mehreren Betrieben zu sammeln hatte, und die unbelastet von der laufenden Betriebsarbeit sich die Zeit nehmen kann, den auftauchenden Problemen nachzugehen, besser als die Betriebsangehörigen selbst erkennen wird, welche Änderungen des bestehenden Zustandes dem Betrieb Vorteile zu bringen versprechen“. ${ }^{29}$

Anders als in den USA oder Großbritannien waren traditionell geführte Familienunternehmen in der Bundesrepublik jedoch nicht leicht von ihrem „Beratungsbedarf“ zu überzeugen. Wie der Einzelberater Walter Schleip I 958 beklagte, werteten Unternehmer das Engagement externer Consultants als Zeichen dafür, „daß sie nicht allein fertig würden“. ${ }^{30}$ Kommerzielle Angebote hatten auch deswegen weniger Zulauf, da korporatistisch organisierte Beratungsstellen der Industrie- und Handelskammern, Wirtschaftsverbände und dem RKW für kleine und mittlere Unternehmen unentgeltlich oder gegen geringe Mitgliederbeiträge Betriebsbegehungen durchführten. ${ }^{3 \mathrm{I}}$ Der Bezeichnung des Unternehmensberaters fehlte zudem ein klar umrissenes Berufsbild. Deutsche Betriebsberater der Nachkriegszeit wie Gerhard Kienbaum stammten üblicherweise aus dem Ingenieurswesen und machten sich erst von Auftrag zu Auftrag mit der Materie der Unternehmensführung vertraut. ${ }^{32}$ Ein ehemaliger Vertreter der Schweizer Knight Wegenstein AG sagte über die Bran-

27 Herbert Strencioch/Bernhard Heitz/Egon Schlobig, Der Wirtschaftsberatungsdienst in den USA. Bericht einer deutschen Studienreise Westberliner Unternehmensberater nach den Vereinigten Staaten von Amerika im Herbst I953. (RKW-Auslandsdienst.) München I957, o. A.

28 Ebd.24.

29 Ebd.o. A.

30 Walter Schleip, ,...ich habe diese Brüder schon einmal dagehabt..." Management Consultants. Ruf und Beratungsgepflogenheit in der Bundesrepublik und im Ausland, in: Junge Wirtschaft 6, I958, 278.

3 I Michael Faust, Consultancies as Actors in Knowledge Arenas. Evidence from Germany, in: Matthias Kipping/Lars Engwall (Eds.), Management Consulting. Emergence and Dynamics of a Knowledge Industry. Oxford 2002, I46-I63. 
chenverhältnisse der I96oer und I970er Jahre, dass „[selbst] ein gelernter Kraftwerksingenieur wie ich [...] mit etwas betriebswirtschaftlichem Zusatzwissen als Consultant reüssieren [konnte]“. ${ }^{33}$

Mit der Festigung einer kommerziellen Consultingbranche ab Mitte der I950er Jahre $^{34}$ begann die staatliche Verwaltung die Dienste nicht nur im Sinne der Wirtschaftsförderung zu stützen, sondern den Gebrauch auch zu eigenen Zwecken zu erwägen ${ }^{35}$. I964 fiel die Bilanz zur „unabhängigen Wirtschaftsberatung in Deutschland“ in einer Dissertation noch vorsichtig aus. Öffentliche Unternehmen nähmen Beratungsdienste zwar in Anspruch, jedoch deutlich seltener als private. ${ }^{36}$ Mehrere verwaltungseigene Einrichtungen ermutigten Behörden jedoch bereits dazu, Unternehmensberater zu engagieren. Die Kommunale Gemeinschaftsstelle für Verwaltungsvereinfachung, die beratend für Gemeinden tätig war, zitierte I966 aus dem „Merkblatt für die Zusammenarbeit mit Unternehmensberatern“ des Deutschen Industrie- und Handelskammertags: „Niemand kann alles wissen. Gerade der kluge und fortschrittlich denkende Unternehmer lässt sich daher beraten." ${ }^{37}$ Zum Verhältnis zwischen verwaltungseigenen Organisationsexperten und Consultants vermerkte der Leiter des Hamburger Organisationsamtes 1977, dass „zwischen internen und externen Beratern keine Konkurrenz besteht, sondern ein Verhältnis der Ergänzung“. ${ }^{38}$ Bisherige Kontakte der öffentlichen Verwaltung zu Beratungsfirmen provozierten weitere Nachfrage, „weil die ,eigenen Berater““, also die Beamten, „überhaupt erst herausfinden, worüber zu beraten ist“. ${ }^{39}$ Die Forecasting-Unternehmen Prognos AG und METRA DIVO beauftragte das Organisationsamt I973 nach

32 Zur Biografie eines der ersten Branchenvertreters der Bundesrepublik siehe Gerhard Kienbaum, Am Anfang war der Rat. Berlin I995.

33 Bernhard Schwoerer, 50 Jahre Unternehmensberatung I954 - 2004, in: TC Team Consult (Hrsg.), Management, Policing and Governance. Festschrift für Bernhard Prestel. Holzkirchen 2004, I 9.

34 Unter anderem indiziert durch die Gründung des ersten deutschen Berufsverbandes, des Bundes Deutscher Unternehmensberater, siehe Heike Borchert-Dietz, BDU-Meilensteine. 50 Jahre Engagement für die Beraterbranche. Bonn 2004.

35 Vgl. Saint-Martins theoretische Annahme, die Praxis des Behörden-Consulting setze eine etablierte Beratungsbranche voraus; Saint-Martin, Managerialist State (wie Anm. 5), 27.

36 Jost Hammerschmidt, Die unabhängige Wirtschaftsberatung in Deutschland. Stand und Ausbaumöglichkeiten unter Berücksichtigung ausländischer Erfahrungen. Nürnberg I964, 74.

37 Staatsarchiv Hamburg, I3 I-I3/433, Mitteilungen der KGSt (wie Anm. 26).

38 Staatsarchiv Hamburg, I 3 I-I II, Nr.434, Notiz Ulrich Becker, Organisationsamt, I 5.4.I 977, I. 39 Ebd. 
eigener Aussage zum „Erwerb des methodischen Rüstzeugs, um selbständig derartige Prognosen [...] vorzunehmen“. ${ }^{40}$

Während einzelne Referate bis Mitte der I970er Jahre Consulting-Untersuchungen nach eigenem Ermessen und zum eigenem Erkenntnisgewinn in Auftrag gaben, wandelten sich die Kräfteverhältnisse im Zuge der vielfach diagnostizierten Strukturbrüche der I 97oer Jahre. ${ }^{4 \mathrm{I}}$ Als Auftraggeber fungierten mit Bundes- und Landesministern oder deren Staatssekretären nun vermehrt die Behördenspitzen. Während die unteren Verwaltungsebenen ihrem Zwecke nach politische Problemlösungsprozesse beratend vorbereiteten, wurden sie nun selbst zum politischen Problem und zum Beratungsgegenstand. Unternehmensberater ersetzten dabei keine Beamten, sondern lieferten Bewertungsstandards, um die Arbeit der nachgeordneten Verwaltung kritisch beurteilen zu können.

Für die Consultants stellte die zunehmende öffentliche Nachfrage zunächst nur eine weitere Verdienstmöglichkeit unter vielen dar. Kleinere Unternehmen witterten die Chance, sich mit dem neuen Marktsegment eine Nische zu sichern. Äußerungen der von Schwarz-Schilling beauftragten Firmen Knight Wendling und Mummert + Partner können exemplarisch dafür herangezogen werden. Im Geschäftsbericht 1975 stellten die Schweizer Berater von Knight Wegenstein (später Knight Wendling) einerseits in Frage, dass „man die vielfältigen Probleme“ der öffentlichen Hand „einfach damit lösen [könne], dass auch der Staat die sogenannten ,bewährten Managementmethoden“ der Wirtschaft einführt“. ${ }^{42}$ Dennoch empfahlen sie Behörden eine betriebswirtschaftliche Gesundungskur. Von vornherein vereinbarte und messbare Ziele sowie ein individuelles Verantwortungsgefühl der Beamten für das Ergebnis der eigenen Handlungen sollten die Lasten der Bürokratie überwinden helfen. Der Geschäftsbericht von I 976 listete „weit verbreitete Symptome“ einer ineffizienten „Verwaltungswirtschaft“ auf. ${ }^{43}$ Zum „[unterentwickelten]

40 Staatsarchiv Hamburg, I3I-2I, Nr.22I6, Vermerk Organisationsamt zu Telefongesprächen mit v. Plotho (Prognos) und Dr. Stöckmann (METRA DIVO) am I8.6.73, 20.6.I973, I.

4I Anselm Doering-Manteuffel/Lutz Raphael, Nach dem Boom. Perspektiven auf die Zeitgeschichte seit I970. Göttingen 2008; Morten Reitmayer/Ruth Rosenberger (Hrsg.), Unternehmen am Ende des „goldenen Zeitalters". Essen 2008.

42 Knight Wegenstein Management Beratung und technische Beratung(Hrsg.), Geschäftsbericht I 975, Zürich I975, I4.

43 Knight Wegenstein Management Beratung und technische Beratung (Hrsg.), Geschäftsbericht I 976, Zürich I976, I6. 
Kostenbewusstsein“ der Mitarbeiter, den unklaren Verantwortungsstrukturen, unpräzisen Zielvorgaben und ungenügenden Ergebniskontrollen gesellte sich die „laienhafte Durchführung von Reorganisationsprojekten“. ${ }^{44}$ „Wie man Reformen entscheidungsreif ausarbeitet, sie grundsätzlich durchsetzt und im Detail realisiert“ bedürfe eines „speziellen Know-[hows]“- das sich die Verfasser augenscheinlich selbst zuschrieben. ${ }^{45}$ Wie seine Wettbewerber nutzte das Unternehmen also das Instrument der öffentlichen Meinungspflege, um ungefragt Problemlagen von Unternehmen und Behörden zu diagnostizieren und deren Abhilfe in Aussicht zu stellen.

Die Anfrage Schwarz-Schillings I 983 beurteilte der Bereichsleiter von Mummert + Partner, Eckard Wohlgehagen, in einem Zeitzeugengespräch als „ein Highlight“ seiner Beraterkarriere. ${ }^{46}$ Als erster Auftrag einer Bundesbehörde hatten die Postprojekte Signalwirkung: „Das war sicherlich einer der Wendepunkte, dass die Bundespost so nachhaltig auf die Berater zuging. “47 Seine Deutung, die Postler „wollten jetzt von der Wirtschaft lernen, jetzt zeigt ihr uns das mal“, blendete mögliche andere taktische Motivationen Schwarz-Schillings aus. ${ }^{48}$ Durch zahlreiche Folgeaufträge, die ihn und seine Teams ganze acht Jahre beschäftigt hielten, betrachtete er sich schließlich selbst als „Leib- und Magenberater“ des Ministers - auch dies möglicherweise eine Überschätzung der Situation. ${ }^{49}$ Dennoch stellten Schwarz-Schillings Berateraufträge als erste groß angelegte Consultingprojekte für eine Bundesbehörde die Weichen für die dauerhafte Etablierung des Behörden-Consultings.

\section{Personalbemessung bei der Deutschen Bundespost: Zwischen Wirtschaftlichkeit und Dienstgüte}

Mitte der I970er Jahre stand die Deutsche Bundespost, wie die sozialliberale Regierung Helmut Schmidts, vor finanzpolitischen Herausforderungen. Auf eine Phase wirtschaftlicher Hochkonjunktur folgte ab 1973 der Abschwung, was sich durch einen Rückgang der Nachfrage insbesondere im Fernmeldewesen ausdrück-

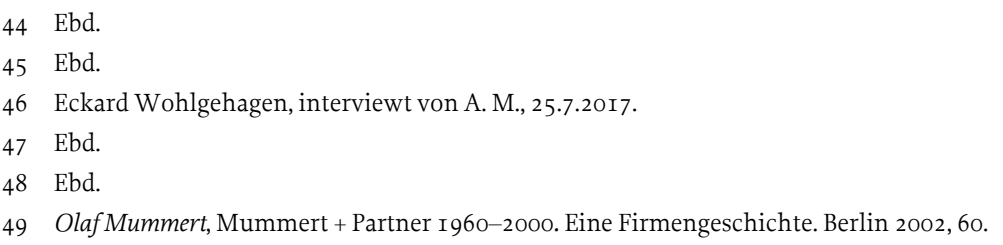


te. ${ }^{50}$ Der Zuwachs an Fernsprechanschlüssen war mit drei bis vier Prozent deutlich hinter den Werten der Vorjahre zurückgeblieben und bescherte den handwerklichen Berufen im Fernmeldewesen weniger Arbeit als erwartet. ${ }^{5 \mathrm{I}}$ Hatte die Bundespost bis dato mit Personalmangel gekämpft, verzeichnete sie nun Personalüberhänge, was den Behördenhaushalt belastete. Die in Zeiten hoher Nachfrage gewonnenen Arbeitskräfte waren entweder unkündbare Beamte oder Auszubildende, die aufgrund der bisherigen Personalpolitik der Bundespost fest mit einer Übernahme rechnen konnten. ${ }^{52}$ Insbesondere das Rationalisierungsschutzabkommen mit den Gewerkschaften von 1972 garantierte jedem Mitarbeiter einen sicheren Arbeitsplatz. ${ }^{53}$ Personaleinsparungen konnten daher nur durch weniger Neueinstellungen erzielt werden. Arbeitswissenschaftliche Berechnungsmethoden sollten diese Bemühungen unterstützen. Laut Personalchef Bernrath würden ab Mitte der r97oer Jahre zunächst „der Aufbau des neuen Personalbemessungssystems und die Anwendung neuer Bemessungswerte zügig vorangetrieben“.54

Eine zentrale Rolle spielte dabei die „Dienstanweisung für die Personalbemessung bei den Ämtern“ (DA Bem Ä). ${ }^{55}$ Das Regelwerk hatte das Bundesministerium für das Post- und Fernmeldewesen I97 I eingeführt, um zu bestimmen, wie viel Personal an den Schaltern der Post- und Fernmeldeämter gebraucht wurde. Zuvor hatten die Oberpostdirektionen auf der mittleren Verwaltungsebene den Personalbedarf geschätzt. ${ }^{56}$ Auf einheitlicher Grundlage der DA Bem Ä sollten nun alle Postund Fernmeldeämter feststellen können, wie viele Arbeitskräfte sie für bestimmte Aufgaben benötigten. Die DA Bem Ä enthielt definierte Bezugseinheiten, die den Umfang bestimmter Tätigkeiten bemaßen, zum Beispiel die „Zahl der zu bearbeiten-

\footnotetext{
50 Hans G. Bernrath/Hubert Lange, Personalpolitik ohne Illusionen, in: Kurt Gscheidle (Hrsg.), Jahrbuch des Postwesens I975. Bad Windsheim I976, 25.

$5 \mathrm{I}$ Ebd.23.

52 Ebd. $27 \mathrm{f}$.

53 Gscheidle, Verbindung (wie Anm. 24), ro8f.

54 Bernrath/Lange, Personalpolitik (wie Anm. 50), 36.

55 Arnold Dohmen, Die Dienstanweisung für die Personalbemessung bei den Ämtern der Deutschen Bundespost (DA Bem Ä), in: Kurt Gscheidle (Hrsg.), Jahrbuch des Postwesens I972. Bad Windsheim I972, I 74268.

56 In Vorbereitung auf die Kriegswirtschaft hatte die wenig aufwendige Beobachtungsmethode I937 das stringentere Leistungszählverfahren nach Taylor ersetzt; Kurt Gscheidle, Die Personalbemessung bei der Deutschen Bundespost, in: ders. (Hrsg.), Jahrbuch der Deutschen Bundespost I982. Bad Windsheim I982, I 2 .
} 
den Briefsendungen, Zugang an Fernsprechhauptanschlüssen, Länge und Stärke der auszulegenden Kabel“. ${ }^{57}$ Jeder Bezugseinheit war mit der durchschnittlich benötigten Zeit je Arbeitsgang ein sogenannter Bemessungswert zugeordnet. Bezugseinheiten und Bemessungswerte sollten in repräsentativen Beispielämtern erhoben und den Ämtern als Anhang zur DA Bem Ä zur Verfügung gestellt werden. I982, elf Jahre nach seiner Einführung, lobte der scheidende Postminister Kurt Gscheidle das Regelwerk als bundesweit einzig in seiner Art.Nur der Verband für Arbeitsstudien REFA könne sich auf ähnlich differenzierte Personalbemessungsmethoden stützen, allerdings im industriellen Fertigungsbereich. ${ }^{58}$

Diese Aussage tätigte Gscheidle in einem Klima, in dem das Bemessungsverfahren seines Hauses unter heftigem Beschuss stand. Der Bundesrechnungshof hatte zunächst begrüßt, dass der Personalbedarf mithilfe der DA Bem Ä „überschaubar, systematisch und möglichst objektiv“ festgestellt werden könne. ${ }^{59}$ Dass die Bemessungsweise der Ämter haushaltswirtschaftlichen Grundsätzen jedoch nicht genügte, hatte das Verwaltungskontrollorgan bereits ab 1976 in seinen jährlich erscheinenden Bemerkungen gegenüber der Bundesregierung deutlich gemacht. Zentrale Bemessungswerte seien erst spät oder gar nicht erstellt worden, sodass viele Postund Fernmeldeämter fünf Jahre nach Einführung der DA Bem Ä trotz der veränderten konjunkturellen Lage noch über keine neuen Werte verfügten. ${ }^{60}$ Die einheitliche Berechnung würde nicht nur verzögert, sondern auch fehlerhaft durchgeführt. Bezugseinheiten seien „nicht zutreffend oder zu ungenau ermittelt“ ${ }^{6 \mathrm{I}}$, und tausende streng genommen nicht benötigte Stellen würden auf die Berechnung aufgeschlagen, um komfortable Arbeitsbedingungen zu gewährleisten. ${ }^{62}$ Das Personalbemessungssystem in seiner damaligen Form beanstandete der Rechnungshof als „ungerecht und unwirtschaftlich“ ${ }^{63}$ und hielt fest:

\footnotetext{
57 Ebd. I4-I6.

58 Ebd.I3.

59 Unterrichtung durch den Bundesrechnungshof. Bemerkungen des Bundesrechnungshofes zur Bundeshaushaltsrechnung (einschließlich der Bundesvermögensrechnung) zum Haushaltsjahr I976, BTDrucksache 8/2 I24. Bonn I978, 75 .

60 Unterrichtung durch den Bundesrechnungshof zum Haushaltsjahr 1974, BT-Drucksache 7/5849. Bonn I976, I00.

6I Unterrichtung durch den Bundesrechnungshof zum Haushaltsjahr I979, BT-Drucksache 9/978. Bonn I 98 I, I48.

62 Ebd.
} 
„Das mit der Personalberechnung vornehmlich verfolgte Ziel, nämlich den

Personalbedarf möglichst frei von Ermessen nach betrieblichen und wirt-

schaftlichen Gesichtspunkten aufgrund anerkannter arbeitswissenschaftli-

cher Methoden zu ermitteln, ist nicht erreicht.“64

Trotz dieser Kritik stieg der Personalbestand im Post- und Fernmeldewesen von I 970 bis I974 um 30000 Stellen pro Jahr an und reduzierte sich auch in der ersten Hälfte der i98oer Jahre nicht. ${ }^{65}$ Wie konnten die Mahnungen des Rechnungshofs bis zum Ende der Regierung Schmidt ungehört verhallen? Zum einen ist zu vermuten, dass das ehemalige Gewerkschaftsvorstandsmitglied Gscheidle den Großteil seiner Amtszeit eher den Forderungen der Belegschaft entgegenkam, als die finanzpolitischen Richtwerte zu priorisieren. ${ }^{66}$ Zum anderen oblag die Auslegung der DA Bem Ä dem guten Willen der mittleren und unteren Verwaltungsebenen. Der Postminister geriet somit sowohl von „oben“ unter Druck als auch - unter anderen Vorzeichen - von „unten“. Die zahlreichen Gewerkschaften und Personalvertretungen im Post- und Fernmeldebereich stellten zähe Verhandlungspartner dar. Seit den frühen I970er Jahren hatten sie so manches Privileg hinsichtlich der Bezahlung, der Sozialleistungen sowie der Arbeitsbedingungen erkämpft. Nun widersetzten sie sich nicht nur einer Einschränkung des Personalbestands, sondern auch der bloßen Neuberechnung des Personalbedarfs. ${ }^{67}$

Dies setzte die Behördenspitze unter Druck, denn das Begehren von „Gewerkschaften und Personalvertretungen deckt sich im allgemeinen nicht mit den unternehmerischen Zielsetzungen zur Anpassung an die Abschwungphase“, wie Personalchef Bernrath bedauerte. ${ }^{68}$ Erwartungen, die noch in der Zeit der Hochkonjunktur genährt worden waren, „machen es besonders schwer, dem Personal die

63 Unterrichtung durch den Bundesrechnungshof zum Haushaltsjahr I977, BT-Drucksache 8/3238. Bonn I979, 64 .

64 Unterrichtung I979 (wie Anm.6I), I47.

65 Unterrichtung I974 (wie Anm.60), 98; Unterrichtung durch den Bundesrechnungshof zum Haushaltsjahr I980, BT-Drucksache 9/2 I08. Bonn I982, I45; Unterrichtung durch den Bundesrechnungshof. Bemerkungen des Bundesrechnungshofes I 985 zur Haushalts- und Wirtschaftsführung, BT-Drucksache ro/ 4367. Bonn I985.

66 Zu Gscheidles Laufbahn als Fernmeldetechniker, stellvertretender Vorsitzender der Deutschen Postgewerkschaft und SPD-Politiker siehe Herbert Uhl, Kurt Gscheidle, in: Udo Kempf/Hans-Georg Merz (Hrsg.), Kanzler und Minister I949-I 998. Biografisches Lexikon der deutschen Bundesregierungen. Wiesbaden 200I, 280-284.

67 Bernrath/Lange, Personalpolitik (wie Anm.50), 29.

68 Ebd.3I. 
Notwendigkeit unternehmerischen Handelns in einer veränderten wirtschaftlichen Lage klarzumachen“. ${ }^{69}$ Als Christian Schwarz-Schilling I982 das Ressort des Post- und Fernmeldewesens übernahm, galt es also, die Arbeitnehmer auf veränderte haushaltspolitische Maßstäbe einzustimmen.

I. „Objektiv und realistisch“, „,neutral und unvoreingenommen“:

Kompetenzstreitigkeiten im Vorfeld der Studie

Während Gscheidle gegenüber dem Bundesrechnungshof stets mit den Verpflichtungen der Deutschen Bundespost als sozialem Arbeitgeber argumentiert hat$t^{70}$, betonte der Unternehmer und ehemalige Mediensprecher der Christdemokraten Schwarz-Schilling ab I983 den Unternehmenscharakter der Behörde. Bei einem Haushaltsvolumen von 66 Milliarden Mark müssten die Entwicklung der Dienste ständig beobachtet werden und „rechtzeitig entsprechende Maßnahmen [eingeleitet werden], um das Unternehmen ,auf Kurs zu halten““. ${ }^{7 \mathrm{I}}$ Bereits als medienpolitischer Sprecher der Christdemokraten hatte sich Schwarz-Schilling für technologische Innovationen im Medien- und Kommunikationsbereich eingesetzt, zuletzt als Teil der Enquête-Kommission „Neue Informations- und Kommunikationstechniken “. ${ }^{72}$ Als neuer Ressortleiter wollte er diese Agenda fortsetzen. Da die Überschüsse im Fernmeldewesen jedoch statt in technologische Modernisierung in die defizitäre „gelbe Post“ mit Brief- und Paketwesen flossen, drang er auf Sparmaßnahmen im Postwesen.

Mit diesem Vorhaben hatte Schwarz-Schilling keinen leichten Stand. Nicht nur die Deutsche Postgewerkschaft und zahlreiche weitere Interessenverbände im Postund Fernmeldewesen stellten sich Rationalisierungsbemühungen entgegen. Auch in den Medien waren Einschränkungen der Postdienste oder gar Gebührenerhöhungen verpönt. In einem Vortrag an der Universität Kiel im Dezember I 983 machte der Minister auf die Diskrepanz zwischen öffentlichem Anspruch und Unternehmenskapazitäten aufmerksam:

\footnotetext{
69 Ebd.3of.

70 Gscheidle, Personalbemessung (wie Anm. 22).

7 I Bundesarchiv (künftig: BArch), B 257/6 I I 26, Sprechzettel für Verwaltungsratssitzung am I6.3.84, I.

72 Siehe Zwischenbericht der Enquete-Kommission „Neue Informations- und Kommunikationstechniken“. BT-Drucksache $9 / 2442$.
} 
„Wenn wir einmal eine Poststelle aufheben wollen, in der in der Woche gerade fünf Stunden Arbeit anfallen, wir aber diejenigen Leute, die dort tätig sind, für zwölf Stunden bezahlen müssen [...], dann ist es wie bei der Bundesbahn: Große Proteste werden veranstaltet, meistens von Leuten, die dieses Amt noch nie gesehen, nie besucht haben.“73

Wirtschaftliches Handeln sei jedoch geboten, um wegweisende Investitionen im Fernmeldewesen zu tätigen. Helmut Kohl bot Schwarz-Schilling in diesen Fragen wenig Rückendeckung. In seiner „Wendeansprache“ von I982 hatte der Kanzler zwar erklärt, er wolle „den Weg freigeben für die Anwendung moderner Techniken und die Entwicklung neuer Technologien, vor allem im Kommunikationswesen“. ${ }^{74}$ Eine „besondere Verantwortung“ komme hierbei der Deutschen Bundespost zu „mit dem geplanten Ausbau der Kabelnetze, der Einführung neuer Dienste sowie der Einbeziehung der Satellitentechnik in ein modernes Kommunikationsnetz“. ${ }^{75}$ Außer weiteren Absichtserklärungen ging vom Kanzler in den folgenden Jahren jedoch keine Initiative für eine veränderte Technologie- oder Finanzpolitik im Post- und Fernmeldewesen aus.

Schwarz-Schilling mobilisierte daher interne und externe Schützenhilfe. Ein Jahr nach Amtsantritt ließ er die Leiter der sieben Abteilungen seines Ministeriums auf einer Klausurtagung zusammenstellen, welche Projekte in ihrem Fachbereich von Unternehmensberatungsfirmen bearbeitet werden könnten. Mögliche Untersuchungen sollten dazu beitragen, die „Leistungs- und Wettbewerbsfähigkeit“ der Post zu sichern und im Fernmeldewesen mit sich wandelnder Kommunikationstechnologie Schritt zu halten. ${ }^{76}$ Die Initiative des neuen Postministers, ausgerechnet kommerzielle Unternehmensberatungsfirmen zu konsultieren, begründete dieser damit, dass diese „neutral“ und „unvoreingenommen“ seien. ${ }^{77}$ Auch bei späteren Folgeaufträgen hieß es, das Engagement einer externen Unternehmensberatungsfirma sei für eine „objektive Analyse [...] und realistische Ergebnisse [...] notwendig“. ${ }^{78}$

73 Christian Schwarz-Schilling, Die Rolle der Deutschen Bundespost im Rahmen der Wirtschaftspolitik der Bundesregierung. Kieler Vorträge gehalten im Institut für Weltwirtschaft an der Universität Kiel. Tübingen 1984,7 .

74 Deutscher Bundestag (Hrsg.), Stenographischer Bericht, 9. WP, I 2 I. Sitzung I 982, 72 I 7.

75 Ebd.

76 BArch, B 257/6II26, Brief von Bundespostminister an den Vorsitzenden des Verwaltungsrates der Deutschen Bundespost, I3.4.I984, 2.

77 BArch, B 257/6 I I 26, Protokoll Verwaltungsratssitzung.

78 BArch, B 257/6I I 29, Vermerk zu Anschlußauftrag an Mummert + Partner, I 7.7.86. 
In besagter Klausurtagung wurden mehrere Geschäftsbereiche der Bundespost als verbesserungswürdig und für externe Beratungsaufträge geeignet identifiziert. Die Bemessungsexperten der Abteilung 3, die für die Personalwirtschaft zuständig war, hatte keine Projektvorschläge eingereicht. Mehrere andere Abteilungen hatten jedoch das Personalbemessungssystem auf die Liste der zu untersuchenden Bereiche gesetzt. Bereits in der Vorentscheidung für oder gegen ein mögliches Projekt kam es zu Kompetenzstreitigkeiten. So sprachen sich die Mitarbeiter von Abteilung 3 gegen eine Untersuchung des Personalbemessungssystems durch externe Unternehmensberater aus, da von einer solchen „u. E. keine praktikablen und betriebsgerechten Verbesserungsvorschläge“ zu erwarten seien. ${ }^{79}$ Das Personalbemessungssystem nach der DA Bem Ä sei „fortlaufend Gegenstand kritischer Vergleiche und von Untersuchungen durch interne wie auch externe Instanzen“.${ }^{80}$ Eigene Erkenntnisse der Abteilung 3, Anregungen anderer Abteilungen sowie die Prüfungsergebnisse von „Revision, Vorprüfungsstellen, BRH, Rechnungsprüfungs- und Haushaltsausschuß des Bundestags“ vermöchten „wegen größerer Sachkunde und Betriebsnähe mit Sicherheit weitaus mehr zu Verbesserungen beizutragen als Hinweise postfremder Unternehmensberater“. ${ }^{8 \mathrm{r}}$

Abteilung 7, deren Vertreter unter anderem mit Erfolgskontrolle und Revision innerhalb der Behörde befasst waren, setzte dem entgegen, für eine Untersuchung seien keine Anwendungskenntnisse bezüglich des gegenwärtigen Bemessungssystems nötig. Vielmehr sei es an der Zeit, „daß aus objektiver Sicht das gesamte Instrumentarium der Personalbemessung einer kritischen Betrachtung unterzogen wird““ ${ }^{82}$ Dazu gehöre neben der „dem System gegenüber notwendige[n] Objektivität des Untersuchers“ auch die „tatsächliche Möglichkeit und Fähigkeit, Vergleiche zwischen Dienstleistungen bei der DBP und solchen der freien Wirtschaft anzustellen“. ${ }^{83}$ Zahlreiche Probleme des gegenwärtigen Systems machten es „notwendig und unabdingbar“, eine Untersuchung durch eine „unvoreingenommene, mit der nötigen Fachkenntnis ausgestattete Unternehmensberatungsfirma anstellen zu lassen“. ${ }^{84}$

Die Zuschreibungen, die hier getätigt wurden, enthielten eine deutliche Kontras- 
tierung zwischen organisationseigenen und organisationsfremden Kompetenzen. Die Fähigkeiten und Eigenschaften, die dem Beamten abgingen, schien der Unternehmensberater in sich zu vereinen: Wirtschaftskompetenz und, wichtiger noch, Unparteilichkeit. Dem Versuch der Beamten aus Abteilung 3, auf der eigenen Sachkenntnis zu beharren, fehlte es an Durchschlagskraft, da er vornehmlich defensiv erschien. Somit wurde das Bemessungssystem auch gegen die Einwände der Personalbewirtschafter in den Katalog mit Untersuchungsvorschlägen aufgenommen.

Nachdem die Abteilungsleiter aus ihrer Erfahrung heraus mögliche Problemstellungen benannt hatten, sollten diese nun mit der Sicht externer Akteure abgeglichen werden. Dazu ließ der Postminister ein separates Auftragsschreiben an sieben Consultingunternehmen versenden. Darunter befanden sich mit der Boston Consulting Group, P. A. Management Consultants, Arthur D. Little und McKinsey \& Company die vier großen und namhaften Unternehmen amerikanischen Ursprungs. Aber auch drei bekannte Unternehmen aus dem deutschsprachigen Raum waren vertreten: Knight Wegenstein, Prognos und Mummert + Partner. ${ }^{85}$ Diese sollten auf Basis eigener Recherchen Schwachstellen im Betriebsablauf feststellen und Projektvorschläge vorlegen.

Das Auftragsschreiben, das sie in Schwarz-Schillings Namen erhielten, problematisierte den „Gegensatz zwischen öffentlichem Auftrag und betriebswirtschaftlicher Führung“, den der Minister öffentlich zum Thema gemacht hatte. ${ }^{86}$ Die Behörde habe „zwar ein betriebswirtschaftliches Instrumentarium mit einem modernen Rechnungswesen, Personalbemessungssystem, mit Statistik, Prognosen, Unternehmensplanung [...] entwickelt“. ${ }^{87}$ Jedoch seien Gewinne und Verluste zwischen dem Post- und dem Fernmeldewesen gegenwärtig stark ungleich verteilt. Als möglicher Auftraggeber formulierte Schwarz-Schilling, welchen Ausweg er anvisierte:

„Es werden also bessere Strategien benötigt, mit denen der ständig anwachsende interne Subventionsbedarf der Postdienste und damit die ökonomischen Behinderungen einer schnellen Ausbreitung der neuen Kommunikationstechniken und -dienste abgebaut werden können. Demgemäß müssen klare und durchsetzbare Zielvorstellungen für die Unternehmenspolitik der

85 BArch, B 257/6 I I 26, Vermerk Stab 30I, Betreff: Unternehmenskonzept für die DBP, I 2.8. I983, 2.

86 BArch, B 257/6 I I 26, Brief des Bundesministers für das Post- und Fernmeldewesen. Betreff: Vorstudie „Unternehmenskonzept für die DBP“" Angebotsanforderung, I.

87 Ebd. 
DBP entwickelt werden, die von den Anforderungen und Bedingungen des Marktes ausgehen.“

Auf diesen Problemaufriss folgte eine lange Liste an Fragen, gruppiert um die Schlagworte Führung, Markt, Rationalisierung, Innovation und Organisation:

"-Sind die vorhandenen verschiedenen Führungsinstrumente (z.B. die mittel- und langfristige Unternehmensplanung) bei der angeführten Doppelstellung der Post ausreichend, um flexibel am Markt operieren zu können?

- Welche Instrumentarien müssen ggf. geschaffen werden, um sicherzustellen, daß die DBP neue Möglichkeiten am Markt schnell erkennt und ggf. umsetzen kann?

- Ist sichergestellt, daß notwendige Innovationsanstöße rechtzeitig erfolgen?

- Werden Rationalisierungsreserven erkannt und ausgeschöpft?

- Wie kann die Dienstleistungs- und Gebührenpolitik so gesteuert werden, daß dem Prinzip der Kostendeckung der einzelnen Dienstzweige besser als im gegenwärtigen Zeitpunkt Rechnung getragen werden kann?

- Bietet die vorhandene Organisation der DBP die geeignetste Voraussetzung dafür, das Unternehmen eigenwirtschaftlich zu führen und Bürger, Wirtschaft und Staat angemessen mit den notwendigen Kommunikationsleistungen zu günstigen Bedingungen zu versorgen?

- Welche Vorteile wären von einem ausgeprägteren Produktmanagement zu erwarten?

- Sind von einer weitgehenden Delegation von Entscheidungsbefugnissen Verbesserungen im internen Dienstbetrieb und positive Auswirkungen auf die Kunden der DBP zu erwarten?“88

Obwohl die Fragestellungen breit und teilweise unverbunden erschienen, hatten sie eines gemeinsam: Sie referierten Schwarz-Schillings Rahmung der Probleme und suggerierten, dass die Bundespost weit kostengünstiger und ertragreicher geführt werden könne. Das Schreiben schloss mit dem Hinweis auf die Rechtsform, an die Post- und Fernmeldewesen gebunden waren. Die Dienste sollten in einem Unternehmen verbleiben. Die Struktur der Deutschen Bundespost in unabhängige Konzerne aufzuteilen, war damit als Lösungsvorschlag ausgeschlossen. Außerdem sollte das Unternehmen weiterhin „an die Grundsätze der Politik der Bundesrepublik 
Deutschland" gebunden sein. ${ }^{89}$ Am öffentlichen Auftrag der Bundespost wurde hier also noch nicht gerüttelt.

Mit Arthur D. Little, Prognos, Mummert + Partner und Knight Wegenstein bekundeten vier Unternehmen Interesse an einem Beratungsprojekt für die Deutsche Bundespost. Sie alle erstellten eine Vorstudie, in denen sie unabhängig von den Abteilungsleitern die drängendsten Unternehmensprobleme identifizieren sollten. Dafür bewegten sich Firmenvertreter von September bis November I 983 im Bundespostministerium, führten Interviews in Mittelbehörden und in Post- und Fernmeldeämtern. ${ }^{90}$ Reaktionen der übrigen drei Unternehmen sind nicht belegt. Es ist aber davon auszugehen, dass das einzige von Schwarz-Schilling formulierte Auswahlkriterium die größeren Firmen von einem Angebot abhielt: Aufträge für die Vorstudien würden „an die günstigsten Anbieter vergeben“. ${ }^{9 \mathrm{I}}$ Die Arbeit mit Behörden erforderte im Vergleich mit privatwirtschaftlichen Unternehmen zudem eine Sensibilität für politische Befindlichkeiten, was gerade Firmen mit hoher Sichtbarkeit zu heikel erschien.

Nach Auswertung der vier Kurzberichte (von denen jeder um die einhundert Seiten umfasste) entschieden die Leiter der sieben Abteilungen gemeinsam mit dem Leiter der Abteilung 902 über den größeren Auftrag. Der Postminister und sein Staatssekretär wurden dabei durch ihre jeweiligen persönlichen Referenten vertreten. Nachdem das Auftragsschreiben unter Schwarz-Schillings Namen verfasst worden war, seien er und der Staatssekretär in Zukunft „nur bei wichtigen Entscheidungen einzuschalten“, wie die Abteilungsleiterrunde festhielt. ${ }^{92}$ Dass die Runde die Auftragsvergabe nicht als „wichtig“ einstufte, indiziert die relative Austauschbarkeit der Firmen und Ergebnisse. Ausschlaggebend erschien vielmehr das Verfahren als solches. Den Zuschlag für die Prüfung des Personalbemessungssystems erhielt im Januar I 984 gegen ein Honorar von I o I o ooo Mark Mummert + Partner. ${ }^{93}$ Mit der öffentlichen Ankündigung der Projekte machte Schwarz-Schilling seinen medialen

\footnotetext{
89 Ebd.3.

90 Aussage von Dietger Hamacher, Projektleiter bei der Knight Wegenstein AG, Archiv der sozialen Demokratie, 5/DPGA400078, HPersR beim BPM. Vermerk über die Besprechung am 4.I I.83 Vorstudie „Unternehmenskonzept für die DBP“, I.

9I BArch, B 257/6II26, Angebotsanforderung (wie Anm. 86), 3.

92 BArch, B 257/6II26, Betreff: Entscheidung über die Vergabe der Aufträge zum Projekt „Unternehmenskonzeption der DBP“, Vermerk, I5.I2.I983, 2.

93 BArch, B 257/6I I 26, Vertrag, 26.I.I984, 5.
} 
und politischen Gegnern deutlich, dass er nicht von seinem restriktiven Haushaltskurs abzuweichen gedenke:

„Um nicht von vornherein einen konzeptionellen Lösungsansatz im Kreuzfeuer der Kritik widerstreitender Interessengruppen zu ersticken und politisch zu ,töten;, habe ich vor kurzem einem namhaften Unternehmensberater den Auftrag erteilt, frei von politischen Einbindungen und Rücksichtnahmen ein Konzept zur Sanierung des Postwesens zu erarbeiten, wie es aus wirtschaftlicher Sicht erforderlich erscheint.“94

\section{Kampf um die Deutungsmacht: Wer ist hier der Experte?}

Ihr geplantes Vorgehen hatten die Berater von Mummert + Partner bereits in ihrem Projektangebot dargelegt. Im Post- und Fernmeldeamt Münster wollten sie anhand des Betriebsablaufs beobachten, ob bestehende Bemessungswerte den tatsächlichen Personalbedarf widerspiegelten. Dafür definierten sie mehrere Untersuchungsbereiche: Abgang, Eingang und die Zustellung von Briefen und Paketen im Falle des Postamtes, sowie Baubezirke, Fernsprechentstörungsstelle und Planungsund Montagestellen für fernmeldetechnische Inneneinrichtungen beim Fernmeldeamt. ${ }^{95}$ Neben der örtlichen Beobachtung sollte das Bemessungssystem nach DA Bem Ä einem Vergleich mit den Postverwaltungen der Schweiz und der Niederlande unterzogen werden. Anhand der erhobenen Daten plante das Team, Schwachstellen in der Personalbemessung zu identifizieren und Verbesserungsvorschläge zu machen.

Wie kompetent das Consultingteam war und wie unparteiisch es sein konnte, verraten zum einen zeitgenössische Selbsteinschätzungen der Berater, zum anderen ihre jeweilige Distanz oder Nähe zu Interessengruppen in der Deutschen Bundespost. Von Personalbemessungsmethoden hatte das Beraterteam laut Mitarbeiter Manner, Diplom-Ingenieur, zunächst „keine Ahnung“. ${ }^{96}$ Auch Projektleiter Wohlgehagen erinnerte sich an den Lernprozess, den das Beraterteam durchlief: „Durch ständiges Befassen mit dieser Materie wurden wir langsam so gut, wie wir zu Beginn

94 Archiv für Christlich-Demokratische Politik, oI-824-6/I, Redemanuskript. Bundespostminister Schwarz-Schilling vor dem Verwaltungsrat, I6.3.I984, 6.

95 BArch, B 257/6II26, Ergebnisprotokoll Tagung der Abteilungsleiter 3 in Freiburg im Breisgau, 6.3.I984.

96 Peter Manner, interviewt von A. M., 2 I.2.20I 8. 
vorgegeben hatten zu sein." ${ }^{97}$ Den Auftrag hatte die Firma nach eigener Aussage durch „forsches Auftreten und vermeintliche Sachkompetenz“ erringen können. ${ }^{98}$ Für die ersten sechs Wochen der zwölfmonatigen Projektlaufzeit hatten die Consultants also, wie bei Beratungsaufträgen in Wirtschaft und Verwaltung üblich, zunächst „desk research“ eingeplant. ${ }^{99}$

Für die Herausbildung der externen Urteilskraft wirkte der interne Sachverstand instrumental. Sieben höhere Postbeamte betreuten das Beraterteam im Rahmen einer „Projektgruppe Personalbemessungssystem“. Darunter befanden sich je zwei Ministerialräte und zwei Postdirektoren, je ein Vertreter vom Post- und vom Fernmeldetechnischen Zentralamt sowie der Münsteraner Oberpostdirektor Walgenbach. Letzterer wurde als „besonders befähigter und in Personalwirtschaftsfragen der DBP erfahrener Beamter“ zur Kontaktperson der Firma ernannt. ${ }^{\text {I0o }}$ Schriftliches Informationsmaterial, das die Beamten den Externen zukommen ließen, sollte diese an den Untersuchungsgegenstand heranführen. Im Gegenzug lieferten Wohlgehagen und seine Mitarbeiter vierteljährliche Zwischenberichte und Zwischenpräsentationen, um über den Fortgang der Untersuchungen zu berichteten. ${ }^{\text {Ior }}$ Die Mitglieder der Projektgruppe sorgten dafür, dass Schwarz-Schillings Erwartungen an den Auftrag genügend Beachtung fanden. So sei „bei allen Vorschlägen insbesondere darauf Wert zu legen, daß eine unangemessene Ausweitung des Personalkörpers vermieden wird“. ${ }^{\text {I02 }}$ Die Forderung nach einer „hinreichenden, aber stets knappen Personalausstattung“ versahen sie mit Dringlichkeit: „I \% Veränderung im Personalbereich bewirkt eine Reduzierung bzw. Erhöhung von jährlichen Kosten um über 200 Mio. DM!“i03

Die enge Zusammenarbeit zwischen Auftraggeber und Auftragnehmer war in der Privatwirtschaft wie in der öffentlichen Verwaltung durchaus typisch. Wie die Firma in ihrem Angebotsschreiben formulierte, müsse der ständige Austausch zwischen Auftraggeber und Auftragnehmer „nicht zwangsläufig zu einer Übereinstim-

\footnotetext{
97 Mummert, Firmengeschichte (wie Anm.49), 60.

98 Ebd.

99 BArch, B 257/6I I26, Vertrag (wie Anm.93), 6.

IOo BArch, B 257/6 I I 26, Vermerk von Referat 326. Personalbemessung der DBP; hier: Untersuchungsauftrag an eine Unternehmensberatungsfirma, 20.I.I984, 2.

IOI BArch, B 257/6II26, Vertrag (wie Anm.93), 2 f.

I02 BArch, B 257/6I I 26, Ergebnisprotokoll (wie Anm. 95), 3.

I03 Ebd.
} 
mung der Meinungen führen“. ${ }^{104}$ Dennoch stellte Wohlgehagen in der Rückschau fest, dass Berater und Beamten in der Projektgruppe „nachher alle in eine Richtung“ dachten. ${ }^{105}$ Dafür mag zuträglich gewesen sein, dass Änderungen im Personalbemessungssystem allein die unteren Verwaltungsschichten betrafen und die Mitglieder der Projektgruppe für ihre Mitarbeit höhere Besoldungsstufen sowie Sachmittelzulagen in Form von Dienstwagen erhielten. Gegenüber dem Minister beschrieben die Consultants seinerzeit die Zusammenarbeit als „uneingeschränkt zufriedenstellend“. ${ }^{\text {106 }}$

Im Gegensatz zu Beamten des höheren Dienstes hatten Gewerkschaften und Personalvertretungen keinen Einfluss auf den Projektverlauf. Der alarmierte Hauptpersonalrat hatte Staatssekretär Florian Ende I 983 zwar das Versprechen abgerungen, „über Aufträge von besonderer Bedeutung“ informiert zu werden. ${ }^{\text {I07 }}$ Als die Personalräte ein paar Monate später jedoch auf weitere Informationen über die Projekte von Knight Wendling und Mummert + Partner drangen, ließ ihnen das Ministerium mitteilen, „daß eine so frühzeitige Einbindung eines Personalratsvertreters in die Untersuchungen nicht zweckmäßig“ sei. ${ }^{108}$ Grund für das Engagement externer Berater sei der Wunsch gewesen, „aus neutraler Sicht zentrale Problemfelder beurteilen zu lassen und nach Möglichkeit unbeeinflußt neue Lösungsvorschläge zu entwi-

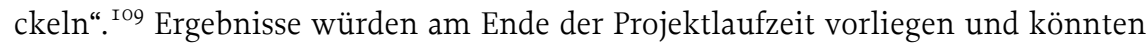
dann auch mit den Personalvertretungen diskutiert werden.

Waren die Gewerkschafter doch einmal zu einer Zwischenpräsentation geladen, wurden ihre Hoffnungen auf Beteiligung enttäuscht. Ein Vermerk Georg Nißls für die Abteilung Personalwirtschaft der Deutschen Postgewerkschaft zeugt davon, dass er sich anlässlich einer Besprechung mit dem Beraterteam wie ein Zaungast vorgekommen war. Eine ursprünglich angesetzte Diskussion von eineinhalb Stunden habe nicht stattgefunden, stattdessen hätten die Erläuterungen der Berater den

\footnotetext{
I04 BArch, B 257/6II26, Mummert \& Partner Unternehmensberatung (Hrsg.), Projektangebot „Personalbemessung der Ämter der DBP“, 27.I.I984, 2.

I05 Interview mit Eckard Wohlgehagen (wie Anm.46), 25.7.20I 7.

I06 BArch, B 257/6 I I 26, Gesprächsprotokoll, Bundespostminister mit Auftragnehmer Mummert + Partner, 9.4.84, 4 .

I07 BArch, B 257/6I I 26, Protokoll der 23. Sitzung des I I. Hauptpersonalrats am 23./24. November I 983. Gespräch mit dem Staatssekretär.

I08 BArch, B 257/6I I 26, Brief von Abteilung 7 an Hauptpersonalrat, 28.2.84, I.

I09 Ebd.
} 
Großteil des dreistündigen Termins eingenommen. Zusätzlich missfallen hatte dem Protokollanten, dass Minister Schwarz-Schilling dem Gespräch „nahezu die ganze Zeit" über beigewohnt hatte und so keine vertraulichen Fragen gestellt werden konnten. ${ }^{\text {II }}$ Auf den Beobachterposten verwiesen blieb den Gewerkschaftsvertretern nur übrig, sich für die Zukunft zu wappnen. Einige der von Mummert + Partner vorgestellten Analysen seien „nicht ungefährlich“, auch wenn das Gutachten erst I 985 fällig würde. ${ }^{\text {II I }}$ Düster schloss Nißl den Vermerk: „Wir werden uns auf einiges gefaßt machen müssen. “ ${ }^{\text {II2 }}$

Im Mai I 985 lud Schwarz-Schilling überraschend und ohne vorherige Absprache mit den Personalvertretungen zu einer Pressekonferenz. Die Projektleiter von Knight Wendling und Mummert + Partner präsentierten die wesentlichen Ergebnisse ihrer jeweiligen Untersuchung und beantworteten an der Seite von Staatssekretär Florian Fragen von Journalisten. Eckard Wohlgehagen führte aus, die Bundespost könne im Postwesen „Personaleinsparungen von deutlich unter Io \%“ sowie im Fernmeldewesen „Zweistellige Prozenteinsparungen“ verwirklichen. ${ }^{\text {II3 }}$ Am folgenden Tag titelte die Süddeutsche Zeitung sogleich: „Gutachter empfehlen der Bundespost Personalabbau und höhere Gebühren; nach Ansicht von Unternehmensberatern kann Zahl der Beschäftigten um etwa zehn Prozent gesenkt werden. “I ${ }^{\text {I }}$

Dies verdeckte, dass der Abschlussbericht von Mummert + Partner keine entscheidungsreifen Vorschläge enthalten hatte. Auftragsgemäß hatten die Consultants den aktuellen Zustand des Bemessungssystems wiedergegeben und diejenigen Probleme erneut aufgeworfen, die der Bundesrechnungshof in der Vergangenheit moniert hatte. Insbesondere nach der Pressekonferenz erschienen quantitative Richtwerte rein schematischer Natur zu sein, so zum Beispiel die im Bericht enthaltene Aufstellung, die Bundespost werde innerhalb der kommenden sechs bis acht Jahre im Postwesen 5 bis 20 Prozent und im Fernmeldewesen 30 bis 35 Prozent Per-

I Io Archiv der sozialen Demokratie, 5/DPGA4IoI38, Vermerk. Mummert \& Partner: Projekt Personalbemessung bei der DBP; Zwischenpräsentation am 26.9.I984, I6.I0.I984, I.

II I Ebd.

II 2 Ebd.

II 3 Archiv der sozialen Demokratie, 5/DPGA400078, Vermerk über die Präsentation der Unternehmensberater Knight Wendling und Mummert \& Partner anläßlich einer Pressekonferenz am I 7.5.85, I0.30 Uhr in Bonn, Hotel Tulpenfeld unter Moderation des Pressesprechers des BPM, Jung, 3.

I 4 Gutachter empfehlen der Bundespost Personalabbau und höhere Gebühren. Nach Ansicht von Unternehmensberatern kann Zahl der Beschäftigten um etwa zehn Prozent gesenkt werden, in: Süddeutsche Zeitung, I8.5.I985, II 4 . 
sonal einsparen können. ${ }^{\text {II }}$ Wie es ein Oberpostdirektor in einer Stellungnahme zum Bericht ausdrückte: „Die eigentliche Gutachtertätigkeit [...] muß jetzt beginnen. “I'6 Darin lieferte sich die Deutsche Postgewerkschaft mit der Projektgruppe Personalbemessungssystem mit dem Tag der Veröffentlichung einen Wettlauf.

Kurt van Haaren, der Vorsitzende der Deutschen Postgewerkschaft, fand deutliche Worte für die Informationspolitik Schwarz-Schillings. Mit der öffentlichen Darstellung, „wir seien postalisch Neandertaler und fernmeldetechnisch ein Entwicklungsland“, habe der Minister den Eindruck erweckt, „Ingenieure, Verwaltungsexperten und wir alle seien unfähig, die Bundespost verantwortlich zu führen“. ${ }^{\text {II7 }}$ Die externe Untersuchung wollte die DPG also als Anschlag auf die Kompetenz der Postverwaltung verstanden wissen und quittierte dies damit, die Befähigung der Berater ihrerseits anzuzweifeln. So attackierte die Gewerkschaftsschrift „Deutsche Post“ die Vorgehensweise von Mummert + Partner als fadenscheinig. Beispiele im Bericht seien „ganz gezielt aufbereitet“ worden, um einen „seriösen Eindruck zu erwecken“. ${ }^{\text {I } 8}$ Die „personaleinsparende[n] ,Verbesserungsvorschläge““ fußten jedoch nach Ansicht der Gewerkschafter auf „zweifelhaften Analysen und [...] verzerrenden internationalen Vergleichen“. ${ }^{\text {II } 9}$ Selbst ein Begleitband zur größten Protestaktion in der Geschichte der Deutschen Postgewerkschaft „Sichert die Post - Rettet das Fernmeldewesen“ nahm Bezug auf das Beratergutachten: „Wir erkennen die eindeutige Absicht, mit geänderten Bemessungselementen nicht mehr den erforderlichen Personalbedarf zu ermitteln und diesen zur Grundlage des Personalhaushalts zu machen.

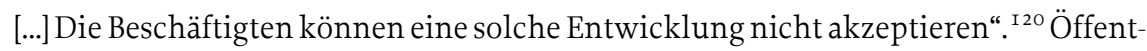
liche Publikationsorgane griffen die nachgereichte Kritik an dem Bericht jedoch kaum auf, sodass dem von Schwarz-Schilling lancierten Narrativ der verschwenderischen Bundespost wenig entgegenstand.

Während die Deutsche Postgewerkschaft das Gutachten in ihrem Sinne auszu-

\footnotetext{
II5 BArch, B 257/6II27, Brief der Projektleitung „Personalbemessungssystem für die Ämter der DBP“ über Abteilungsleiter 3 an Staatssekretär, 30.9.1985, 2.

II6 BArch, B 257/6I I 28, Stellungnahme zum Abschlußbericht der Unternehmensberatung Mummert + Partner, OPDir Dipl.-Ing. Werner Pohl, 28.8.85, 5.

I 7 Kurt van Haaren, Dokumentation. Sichert die Post - Rettet das Fernmeldewesen. o. O. I986, 683.

II 8 Personalbemessung (wie Anm.6) in: Deutsche Post, 20.6.I985, I2.

II9 Ebd.

I20 Mit einer starken DPG. Sichere Perspektiven für Bundespost und Arbeitnehmer. Frankfurt am Main I986, I 26.
} 
deuten versuchte, bat die Projektgruppe Personalbemessung die Referatsleiter für Personalwirtschaft bei den Oberpostdirektionen um ihre fachliche Beurteilung der Beratervorschläge. Ähnlich wie die Gewerkschafter fühlte sich auch die mittlere Verwaltungsebene genötigt, die Daseinsberechtigung der eigenen Bemessungsfachleute zu bekräftigen. Unglaubwürdig wäre es gewesen, die von Mummert + Partner identifizierten, weithin bekannten Problemquellen als irrelevant zu verklären. Die Beanstandungen jedoch nur zu bestätigen, hätte die interne Expertise redundant erscheinen lassen. Die Personalwirtschafter billigten daher die generelle Stoßrichtung des Gutachtens, nur um den Großteil ihrer Rückmeldungen einer ausführlichen Methodenkritik zu widmen. Die Mängelanalyse des Bemessungssystems sei soweit vertraut, bleibe aber vage und lasse ein „Rezept“ zur Behebung der Schwierigkeiten vermissen. ${ }^{\text {I2I }}$ Der von Mummert + Partner angestellte internationale Vergleich sei außerdem nicht zu werten, da die Rahmenbedingungen der Schweizer und niederländischen Postverwaltungen sich erheblich von denen der Bundespost unterschieden. Darüber hinaus verkannten die Berater aus Sicht der Beamten, dass Stellenreduzierungen nicht freiheraus verordnet werden konnten, sondern mit den Personalvertretungen abgestimmt werden mussten. Immerhin: Den Bericht ziere ein „profihaftes Layout“. ${ }^{\text {I22 }}$ Die Gliederung sei übersichtlich, die Anlagen farbig und der „breite Rand neben dem Text“ eigne sich für persönliche Notizen. ${ }^{\text {I23 }}$

Die vielfältige Kritik, die die Personalwirtschafter der Oberpostdirektionen an Mummert + Partners Schriftstück geübt hatten, blieb in der Bilanz der Projektgruppe lediglich implizit. Schließlich war es im Sinne des Ministers, die Notwendigkeit des kostspieligen Unterfangens gegenüber dem Bundesrechnungshof und dem Finanzministerium vertreten zu können. Nach mehrmonatiger Auswertung des I35 Seiten starken Berichts urteilten Walgenbach und sein Kreis: „Die Analyse des Istzustands [des Bemessungssystems] ist weitgehend richtig; die aufgezeigten Mängel müssen deshalb - bis auf einige Ausnahmen - anerkannt werden. " ${ }^{224}$ Positiv hob die Projektgruppe hervor, dass die Firma den „grundsätzlichen Ansatz“ des Bemessungs-

I 2 I BArch, B 257/6 I I 28, Schlußbericht der Unternehmensberatung Mummert + Partner, POR Dipl.-Ing. Rainer Friedrichowitz, 28.8.1985, 3. Ähnlich: BArch, B 257/6I I28, Postoberrat Dipl.-Ing. Perske, 2.9.I985; BArch, B 257/6 I I 28, Stellungnahme zum Schlußbericht der Unternehmensberatung Mummert + Partner, POR Dipl-Ing. Michael Strueß, 8.9.1985.

I 2 BArch, B 257/6I I 28, Schlußbericht, POR Friedrichowitz (wie Anm. I 2 I), I.

I23 Ebd.

I24 BArch, B 257/6I I 27, Brief der Projektleitung (wie Anm. I I5), 2. 


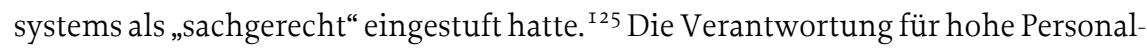
bestände wurde damit erneut den unteren Verwaltungsebenen zugeschrieben - ein schriftlicher Beleg, der den Minister entlastete und der sich in das Bild einfügte, das die Berichterstattung bereits gezeichnet hatte. Unberücksichtigt blieb dabei die Tatsache, dass Walgenbachs Projektgruppe selbst die verheißungsvollen Zahlenwerke der Berater intern ebenfalls skeptisch beurteilte. Eine Senkung des Personalbestands, so die Abwägung der Beamten, habe „in absehbarer Zeit negative Folgen auf den Arbeitsmarkt“, aber „auf Dauer positive Folgen für die Kunden, die Gebührenpolitik und die betriebliche Abwicklung“. ${ }^{126}$ Die genannten Einspargrößen seien jedoch „weder durch die Feststellungen im Bericht belegt, noch entsprechen sie realistischen Einschätzungen.“" ${ }^{27}$

Ein Redemanuskript des Parlamentarischen Staatssekretärs Rawe für eine Fragestunde im Deutschen Bundestag I 986 zeugt davon, dass die Behördenspitze auf Kritik gefasst war. Darin war notiert, dass „allein schon die innerhalb und außerhalb der DBP geführte Diskussion“ über das Gutachten dieses „bezahlt gemacht“ habe. ${ }^{\text {I28 }}$ Die Firma habe bekannte Punkte aufgeworfen, aber auch „zum Teil“ konzeptionell neue Vorschläge gemacht, die sich positiv auf das Bemessungsverfahren auswirken könn-

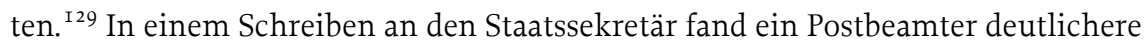
Worte für den eigentlichen Erfolg der Untersuchungen aus Sicht der Unternehmensspitze:

„Die zwar schon vorgesehene, aber schwierige Durchsetzung der Reduzierung [des Personals] wurde zweifellos beschleunigt, da mit dem Gutachten der Personalvertretung und den Gewerkschaften viel ,Wind aus den Segeln` genommen war.“ ‘30

\footnotetext{
I25 Ebd.

I26 Ebd.3.

I27 Ebd.2.

I28 BArch, B 257/6I I28, Antwortvorschlag: Mündliche Fragestunde des Dt. Bundestages am 22./23.I.86, I.

I29 Ebd.

I30 BArch, B 257/6I I29, Brief von Abteilung 2a an Staatssekretär. Antwort auf Schreiben von Staatssekretär vom I3.8.86, 2.I0.I986.
} 


\section{Fazit: Unternehmensberater als Überbringer unbequemer Nachrichten}

Im Kampf zwischen Selbsterhaltung und Rationalisierung der Deutschen Bundespost rangen Anfang der I98oer Jahre zwei Arten entscheidungsvorbereitender Wissensbestände miteinander: der gebietsbezogene Sachverstand der Beamten, der auf langjähriger Erfahrung beruhte und auf die öffentliche Daseinsvorsorge sowie auf Arbeitnehmerinteressen ausgerichtet war; und die ad hoc mobilisierte Intervention der Berater, die sich auf übergeordnete Organisationsprinzipien berief und das öffentliche Unternehmen unter wirtschaftlichen Vorzeichen betrachtete. Unter dem Deckmantel von Kompetenzstreitigkeiten ging es um die Fragen, wie viel staatliche Leistungen kosten durften und welcher Grad an Dienst- und Betriebsgüte gerechtfertigt war.

Der Expertenstatus der Fachbeamten bei der Deutschen Bundespost wandelte sich in der Konfrontation mit Mummert + Partner auf ambivalente Art.Durch das Engagement externer Berater entzogen Schwarz-Schilling und sein Führungsstab den Beamten der Ministeriumsabteilung Personalwirtschaft Vertrauen. Dennoch trugen sie ihnen auf, die Gutachtervorschläge auf ihre Tauglichkeit zu prüfen. Ihre Sachkunde schien der Postminister seinen Untergebenen also nicht abzusprechen; wohl aber ihre Fähigkeit, angesichts eigener Ansprüche an die Dienst- und Betriebsgüte Objektivität walten zu lassen. Um ihr Gesicht zu wahren, blieb den Bemessungsspezialisten wenig anderes übrig, als die festgestellten Mängel als altbekannt abzutun und das Gutachten für mangelnde Exaktheit und damit fehlende Fachkenntnis zu kritisieren. Somit hatte die Verwaltungsspitze keine Handlungsdirektive gewonnen. Die Firma Mummert + Partner nahm sich der staatlichen Ratlosigkeit weiterhin bereitwillig an, da daraus bis 1988 zusätzliche Projekte für die Deutsche Bundespost resultierten und die Spitzen anderer Bundes- und Landesbehörden ebenfalls auf ihre Dienstleistung aufmerksam wurden. ${ }^{\text {I3I }}$ Die Konzentration einzelner Firmen auf den öffentlichen Sektor ließ mit der Zeit das Bild der unbedarften Gene-

I3 I Darunter das Land Nordrhein-Westfalen, das von I99I bis 999 in einer großangelegten Aktion Organisationsuntersuchungen in allen Landesbehörden anordnete. Mummert + Partner erstellten hier unter anderem eine Studie zur Lehrerarbeitszeit: Mummert + Partner Unternehmensberatung (Hrsg.), Untersuchung zur Ermittlung, Bewertung und Bemessung der Arbeitszeit der Lehrerinnen und Lehrer im Land Nordrhein-Westfalen. Düsseldorf I999. 
ralisten verblassen. Mit jedem Auftrag sammelten die Beratungsfirmen Referenzen, die ihre wachsende Kenntnis des rechtlichen Rahmens staatlicher Organisationen belegen und sie von Wettbewerbern abheben sollten.

Die nähere Betrachtung hat gezeigt, dass Mummert + Partner nicht so „objektiv“ und „neutral“ vorgingen, wie von Schwarz-Schilling vorgegeben. Bewusst orientierte das Consultingteam die Untersuchungsanordnung an den Vorgaben des Auftraggebers und ließ die Ergebnisdarstellung mit dessen Zielsetzung harmonieren. Die mediale Aufbereitung der Empfehlungen machte die Kompetenzzuschreibung jedoch wirksam und verschaffte dem Postminister in zweifacher Hinsicht taktische Vorteile. Zum einen konnte er sich die grundsätzliche Eignung des Systems gegenüber dem Bundesrechnungshof bescheinigen lassen und demonstrieren, dass er um eine wirtschaftlichere Haushaltsführung bemüht war. Zum anderen wurden lange bekannte Defizite des Personalbemessungssystems einer breiten Öffentlichkeit dargelegt, was die Belegschaftsproteste gegen Stellenstreichungen schwächte. Unternehmensberater lieferten politischen Entscheidungsträgern in den I980er Jahren also nicht in erster Linie Expertenwissen, sondern vor allem Begründungsnarrative für einen nachdrücklicher verfolgten Konsolidierungskurs. 
